\title{
Compact orbit spaces in Hilbert spaces and limits of edge-colouring models
}

\author{
Guus Regts, Alexander Schrijver \\ University of Amsterdam, Netherlands
}

\section{A R T I C L E I N F O}

Article history:

Available online 5 August 2015

\begin{abstract}
A B S T R A C T
Let $G$ be a group of orthogonal transformations of a real Hilbert space $H$. Let $R$ and $W$ be bounded $G$-stable subsets of $H$. Let $\|\cdot\|_{R}$ be the seminorm on $H$ defined by $\|x\|_{R}:=\sup _{r \in R}|\langle r, x\rangle|$ for $x \in H$. We show that if $W$ is weakly compact and the orbit space $R^{k} / G$ is compact for each $k \in \mathbb{N}$, then the orbit space $W / G$ is compact when $W$ is equipped with the norm topology induced by $\|\cdot\|_{R}$.

As a consequence we derive the existence of limits of edgecolouring models which answers a question posed by Lovász. It forms the edge-colouring counterpart of the graph limits of Lovász and Szegedy, which can be seen as limits of vertex-colouring models.

In the terminology of de la Harpe and Jones, vertex- and edgecolouring models are called 'spin models' and 'vertex models' respectively.
\end{abstract}

(c) 2015 Elsevier Ltd. All rights reserved.

\section{Introduction}

In a fundamental paper, Lovász and Szegedy [10] showed that the collection of simple graphs fits in a natural way in a compact metric space $W$ that conveys several phenomena of extremal and probabilistic graph theory and of statistical mechanics. In particular, a limit behaviour of graphs can be derived.

The elements of $W$ are called graphons, as generalization of graphs, but they can also be considered to generalize the vertex-colouring models, or 'spin models' in the sense of de la Harpe and Jones [2]. In this context, the partition functions of spin models form a compact space. In the present paper, we investigate to what extent edge-colouring models, or 'vertex models' in the terminology of [2],

E-mail address: G.Regts@uva.nl (G. Regts). 
behave similarly. Indeed, the edge-colouring models form a dense subset in a compact space, and thus we obtain limits of edge-colouring models. This solves a problem posed by Lovász [8].

To obtain these results, we prove a general theorem on compact orbit spaces in Hilbert space, that applies to both vertex and edge-colouring models. This compactness theorem uses and extends theorems of Lovász and Szegedy [11] on Szemerédi-like regularity in Hilbert spaces.

For background on graph limits we also refer to the recent book of Lovász [9]. Partition functions of edge-colouring models with a finite number of states were characterized by Szegedy [14] and Draisma, Gijswijt, Lovász, Regts, and Schrijver [3].

\section{Formulation of results}

In this section we describe our results, giving proofs in Sections 2.1,2.2, and 2.3. Throughout, for any Hilbert space $H, B(H)$ denotes the closed unit ball in $H$. Moreover, measures are Lebesgue measures.

Compact orbit spaces in Hilbert spaces. Let $H$ be a real Hilbert space and let $R$ be a bounded subset of $H$. Define a seminorm $\|\cdot\|_{R}$ and a pseudometric ${ }^{1} d_{R}$ on $H$ by, for $x, y \in H$ :

$$
\|x\|_{R}:=\sup _{r \in R}|\langle r, x\rangle| \text { and } d_{R}(x, y):=\|x-y\|_{R} .
$$

In this paper, we use the topology induced by this pseudometric only if we explicitly mention it, otherwise we use the topology induced by the usual Hilbert norm $\|$.$\| .$

A subset $W$ of $H$ is called weakly compact if it is compact in the weak topology on $H$. Note that for any $W \subseteq H$ (cf. [7]):

$W$ closed, bounded, and convex $\Rightarrow W$ weakly compact $\Rightarrow W$ bounded.

Let $G$ be a group acting on a topological space $X$. Then the orbit space $X / G$ is the quotient space of $X$ taking the orbits of $G$ as classes. A subset $Y$ of $X$ is called $G$-stable if $g \cdot y \in Y$ for each $g \in G$ and $y \in Y$.

Our first main theorem (which we prove in Section 2.1) is:

Theorem 1. Let $H$ be a Hilbert space and let $G$ be a group of orthogonal transformations of $H$. Let $W$ and $R$ be $G$-stable subsets of $H$, with $W$ weakly compact and $R^{k} / G$ compact for each $k$. Then $\left(W, d_{R}\right) / G$ is compact.

Application to graph limits and vertex-colouring models. As a consequence of Theorem 1 we now first derive the compactness of the graphon space, which was proved by Lovász and Szegedy [11]. Let $H=L^{2}\left([0,1]^{2}\right)$, the set of all square integrable functions $[0,1]^{2} \rightarrow \mathbb{R}$. Let $R$ be the collection of functions $\chi^{A} \times \chi^{B}$, where $A$ and $B$ are measurable subsets of $[0,1]$ and where $\chi^{A}$ and $\chi^{B}$ denote the incidence functions of $A$ and $B$. Let $S_{[0,1]}$ be the group of measure space automorphisms of [0,1]. The group $S_{[0,1]}$ acts naturally on $H$. Moreover, $R^{k} / S_{[0,1]}$ is compact for each $k$. (This can be derived from the fact that for each measurable $A \subseteq[0,1]$ there is a $\pi \in S_{[0,1]}$ such that $\pi(A)$ is an interval up to a set of measure 0 (cf. [12]).)

Let $W$ be the set of [0,1]-valued functions $w \in H$ satisfying $w(x, y)=w(y, x)$ for all $x, y \in[0,1]$. Then $W$ is a closed bounded convex $S_{[0,1]}$-stable subset of $H$. So by Theorem $1,\left(W, d_{R}\right) / S_{[0,1]}$ is compact. The elements of $W$ are called graphons, where two elements $w, w^{\prime}$ of $W$ are assumed to be the same graphon if $w^{\prime}=g \cdot w$ for some $g \in S_{[0,1]}$. Therefore one may say that the graphon space is compact with respect to $d_{R}$.

In the context of de la Harpe and Jones [2], graphons can be considered as 'spin models' (with infinitely many states). For any $w \in W$, the partition function $\tau(w)$ of $w$ is given by, for any graph $F$ :

$$
\tau(w)(F):=\int_{[0,1]^{V(F)}} \prod_{u v \in E(F)} w(x(u), x(v)) d x .
$$

\footnotetext{
1 A seminorm is a norm except that nonzero elements may have norm 0 . A pseudometric is a metric except that distinct points may have distance 0 . One can turn a pseudometric space into a metric space by identifying points at distance 0 , but for our purposes it is notationally easier and sufficient to maintain the original space. Notions like convergence pass easily over to pseudometric spaces, but limits need not be unique.
} 
Let $\mathcal{F}$ denote the collection of simple graphs. Lovász and Szegedy [10] showed that $\tau:\left(W, d_{R}\right) \rightarrow \mathbb{R}^{\mathcal{F}}$ is continuous (here the restriction to simple graphs is necessary). Since $\left(W, d_{R}\right) / S_{[0,1]}$ is compact and since $\tau$ is $S_{[0,1]}$-invariant, the collection of functions $f: \mathcal{F} \rightarrow \mathbb{R}$ that are partition functions of graphons is compact. Hence each sequence $\tau\left(w_{1}\right), \tau\left(w_{2}\right), \ldots$ of partition functions of graphons $w_{1}, w_{2}, \ldots$, such that $\tau\left(w_{i}\right)(F)$ converges for each $F$, converges to the partition function $\tau(w)$ of some graphon $w$.

Since simple graphs can be considered as elements of $W$ (by considering their adjacency matrix as element of $W$ ), this also gives a limit behaviour of simple graphs.

Application to edge-colouring models. We next show how Theorem 1 applies to the edge-colouring model (also called vertex model). For this, it will be convenient to use a different, but universal model of Hilbert space. Let $C$ be a finite or infinite set, and consider the Hilbert space $H:=\ell^{2}(C)$, the set of all functions $f: C \rightarrow \mathbb{R}$ with $\sum_{c \in C} f(c)^{2}<\infty$, having norm $\|f\|:=\left(\sum_{c \in C} f(c)^{2}\right)^{1 / 2}$. (Each Hilbert space is isomorphic to $\ell^{2}(C)$ for some $C$.) Following de la Harpe and Jones [2], any element of $\ell^{2}(C)$ is called an edge-colouring model, with state set (or colour set) $C$.

Define for each $k=0,1, \ldots$ :

$$
H_{k}:=\ell^{2}\left(C^{k}\right) .
$$

The tensor power $\ell^{2}(C)^{\otimes k}$ embeds naturally in $\ell^{2}\left(C^{k}\right)$, and the orthogonal group $O(H)$ of $H$ acts naturally on $H_{k}$. Define moreover

$$
R_{k}:=\left\{r_{1} \otimes \cdots \otimes r_{k} \mid r_{1}, \ldots, r_{k} \in B(H)\right\} \subseteq H_{k} .
$$

Again, let $\mathcal{F}$ be the collection of simple graphs. As usual, $H_{k}^{S_{k}}$ denotes the set of elements of $H_{k}$ that are invariant under the natural action of $S_{k}$ on $H_{k}$. Define the function

$$
\pi: \prod_{k=0}^{\infty} H_{k}^{S_{k}} \rightarrow \mathbb{R}^{\mathcal{F}} \text { by } \pi(h)(F):=\sum_{\phi: E(F) \rightarrow C} \prod_{v \in V(F)} h_{\operatorname{deg}(v)}(\phi(\delta(v)))
$$

for $h=\left(h_{k}\right)_{k \in \mathbb{N}} \in \prod_{k \in \mathbb{N}} H_{k}^{S_{k}}$ and $F \in \mathcal{F}$. Here $\operatorname{deg}(v)$ denotes the degree of $v$. Moreover, $\delta(v)$ is the set of edges incident with $v$, in some arbitrary order, say $e_{1}, \ldots, e_{k}$, with $k:=\operatorname{deg}(v)$. Then $\phi(\delta(v)):=\left(\phi\left(e_{1}\right), \ldots, \phi\left(e_{k}\right)\right)$ belongs to $C^{k}$. (So $\phi(\delta(v))$ may be viewed as the set of colours 'seen' from $v$.) For (6), the order chosen is irrelevant, as $h_{k}$ is $S_{k}$-invariant.

The function $\pi(h): \mathcal{F} \rightarrow \mathbb{R}$ can be considered as the partition function of the edge-colouring model $h$. It is not difficult to see that $\pi$ is well-defined, and continuous if we take the usual Hilbert metric on each $H_{k}$, even if we replace $\mathcal{F}$ be the collection of all graphs without loops (cf. (14)). For simple graphs it remains continuous on $\prod_{k} B_{k}$ where $B_{k}:=B\left(H_{k}^{S_{k}}\right)$ if we replace for each $k$ the Hilbert metric on $B_{k}$ by $d_{R_{k}}$ :

Theorem 2. $\pi$ is continuous on $\prod_{k \in \mathbb{N}}\left(B_{k}, d_{R_{k}}\right)$.

This is proved in Section 2.2, while in Section 2.3 we derive from Theorem 1:

Theorem 3. $\left(\prod_{k=0}^{\infty}\left(B_{k}, d_{R_{k}}\right)\right) / O(H)$ is compact.

Now $\pi$ is $O(H)$-invariant. This follows from the facts that $\ell^{2}\left(C^{k}\right)$ is the completion of $\ell^{2}(C)^{\otimes k}$ and that $O(H)$-invariance is direct if each $h_{k}$ belongs to $\ell^{2}(C)^{\otimes k}$. Hence Theorem 3 implies:

Corollary 3a. The image $\pi\left(\prod_{k} B_{k}\right)$ of $\pi$ is compact.

This implies:

Corollary 3b. Let $h^{1}, h^{2}, \ldots \in \prod_{k} B_{k}$ be such that for each simple graph $F, \pi\left(h^{i}\right)(F)$ converges as $i \rightarrow \infty$. Then there exists $h \in \prod_{k} B_{k}$ such that for each simple graph $F, \lim _{i \rightarrow \infty} \pi\left(h^{i}\right)(F)=\pi(h)(F)$. 
As $\ell^{2}\left(C^{\prime}\right)$ embeds naturally in $\ell^{2}(C)$ if $C^{\prime} \subseteq C$, all edge-colouring models with any finite number of states embed in $\ell^{2}(C)$ if $C$ is infinite. So the corollary also describes a limit behaviour of finite-state edge-colouring models, albeit that the limit may have infinitely many states.

The corollary holds more generally for sequences in $\prod_{k} \lambda_{k} B_{k}$, for any fixed sequence $\lambda_{0}, \lambda_{1}, \ldots$ $\in \mathbb{R}$.

We do not know if the quotient function $\pi / \sim:\left(\prod_{k} B_{k}\right) / \sim \rightarrow \mathbb{R}^{\mathcal{F}}$ is one-to-one, where $\sim$ is the equivalence relation on $\prod_{k} B_{k}$ with $h \sim h^{\prime}$ whenever $h^{\prime}$ belongs to the closure of the $O(H)$-orbit of $h$. (For finite $C$ and $\mathcal{F}$ replaced by the set of all graphs, this was proved in [13].) The analogous result for vertex-colouring models (i.e., graph limits) was proved by Borgs, Chayes, Lovász, Sós, and Vesztergombi [1].

\subsection{Proof of Theorem 1}

In this section we give a proof of Theorem 1.

Proposition 1. Let $H$ be a Hilbert space and let $R, W \subseteq H$ with $R$ bounded and $W$ weakly compact. Then $\left(W, d_{R}\right)$ is complete.

Proof. Let $a_{1}, a_{2}, \ldots \in W$ be a Cauchy sequence with respect to $d_{R}$. We must show that it has a limit in $W$, with respect to $d_{R}$. As $W$ is weakly compact, it has the Bolzano-Weierstrass property; that is, there is a point $a$ such that each weak neighbourhood of $a$ contains infinitely many terms of $a_{1}, a_{2}, \ldots$ Then $a$ is a required limit, that is, $\lim _{n \rightarrow \infty} d_{R}\left(a_{n}, a\right)=0$. For choose $\varepsilon>0$. As $a_{1}, a_{2}, \ldots$ is a Cauchy sequence with respect to $d_{R}$, there is a $p$ such that $d_{R}\left(a_{n}, a_{m}\right)<\frac{1}{2} \varepsilon$ for all $n, m \geq p$. For each $r \in R$, as each weak neighbourhood of $a$ contains infinitely many terms of $a_{1}, a_{2}, \ldots$, there is an $m \geq p$ with $\left|\left\langle r, a_{m}-a\right\rangle\right|<\frac{1}{2} \varepsilon$. This implies for each $n \geq p$ :

$$
\left|\left\langle r, a_{n}-a\right\rangle\right| \leq\left|\left\langle r, a_{n}-a_{m}\right\rangle\right|+\left|\left\langle r, a_{m}-a\right\rangle\right|<\varepsilon .
$$

So $d_{R}\left(a_{n}, a\right) \leq \varepsilon$ if $n \geq p$.

Let $G$ be a group acting on a pseudometric space $(X, d)$ that leaves $d$ invariant. Define a pseudometric $d / G$ on $X$ by, for $x, y \in X$ :

$$
(d / G)(x, y)=\inf _{g \in G} d(x, g \cdot y) .
$$

As $d$ is $G$-invariant, $(d / G)(x, y)$ is equal to the distance of the $G$-orbits $G \cdot x$ and $G \cdot y$. Any two points $x, y$ on the same $G$-orbit have $(d / G)(x, y)=0$. If we identify points of $(X, d / G)$ that are on the same orbit, the topological space obtained is homeomorphic to the orbit space $(X, d) / G$ of the topological space $(X, d)$. Note that being compact or not is invariant under such identifications.

Proposition 2. If $(X, d)$ is a complete metric space, then $(X, d / G)$ is complete.

Proof. Let $a_{1}, a_{2}, \ldots \in X$ be a Cauchy sequence with respect to $d / G$. Then it has a subsequence $b_{1}, b_{2}, \ldots$ such that $(d / G)\left(b_{k}, b_{k+1}\right)<2^{-k}$ for all $k$. Let $g_{1}=1 \in G$. If $g_{k} \in G$ has been found, let $g_{k+1} \in G$ be such that $d\left(g_{k} b_{k}, g_{k+1} b_{k+1}\right)<2^{-k}$. Then $g_{1} b_{1}, g_{2} b_{2}, \ldots$ is a Cauchy sequence with respect to $d$. Hence it has a limit, say $b$. Then $\lim _{k \rightarrow \infty}(d / G)\left(b_{k}, b\right)=0$, implying $\lim _{n \rightarrow \infty}(d / G)\left(a_{n}, b\right)=0$.

Let $H$ be a Hilbert space and let $R \subseteq H$. For any $k \geq 0$, define

$$
Q_{k}=\left\{\lambda_{1} r_{1}+\cdots+\lambda_{k} r_{k} \mid r_{1}, \ldots, r_{k} \in R, \lambda_{1}, \ldots, \lambda_{k} \in[-1,+1]\right\} .
$$

For any pseudometric $d$, let $B_{d}(Z, \varepsilon)$ denote the set of points at distance at most $\varepsilon$ from $Z$. The following is a form of 'weak Szemerédi regularity'. (cf. Lemma 4.1 of Lovász and Szegedy [11], extending results of Frieze and Kannan [6] and Fernandez de la Vega, Kannan, Karpinski, and Vempala [5]-the method is essentially that of [6]):

Proposition 3. If $R \subseteq B(H)$, then for each $k \geq 1$ :

$$
B(H) \subseteq B_{d_{R}}\left(Q_{k}, 1 / \sqrt{k}\right) .
$$


Proof. Choose $a \in B(H)$ and set $a_{0}:=a$. If, for some $i \geq 0, a_{i}$ has been found, and $d_{R}\left(a_{i}, 0\right)>1 / \sqrt{k}$, choose $r \in R$ with $\left|\left\langle r, a_{i}\right\rangle\right|>1 / \sqrt{k}$. Define $a_{i+1}:=a_{i}-\left\langle r, a_{i}\right\rangle$. Then, with induction,

$$
\left\|a_{i+1}\right\|^{2}=\left\|a_{i}\right\|^{2}-\left\langle r, a_{i}\right\rangle^{2}\left(2-\|r\|^{2}\right) \leq\left\|a_{i}\right\|^{2}-\left\langle r, a_{i}\right\rangle^{2} \leq\left\|a_{i}\right\|^{2}-1 / k \leq 1-(i+1) / k .
$$

Moreover, as $\left\langle r, a_{i}\right\rangle \in[-1,+1]$, we know by induction that $a-a_{i} \in Q_{i}$.

$\operatorname{By}(11)$, as $\left\|a_{i+1}\right\|^{2} \geq 0$, the process terminates for some $i \leq k$. For this $i$ one has $d_{R}\left(a_{i}, 0\right) \leq 1 / \sqrt{k}$. Hence, as $Q_{i} \subseteq Q_{k}, d_{R}\left(a, Q_{k}\right) \leq d_{R}\left(a, Q_{i}\right) \leq d_{R}\left(a, a-a_{i}\right)=d_{R}\left(a_{i}, 0\right) \leq 1 / \sqrt{k}$.

Proof of Theorem 1. Observe that $R$ is bounded as $R / G$ is compact. So we can assume that $R, W \subseteq$ $B(H)$.

By Propositions 1 and $2,\left(W, d_{R} / G\right)$ is complete. So it suffices to show that $\left(W, d_{R} / G\right)$ is totally bounded; that is, for each $\varepsilon>0, W$ can be covered by finitely many $d_{R} / G$-balls of radius $\varepsilon$ (cf. [4]).

Set $k:=\left\lceil 4 / \varepsilon^{2}\right\rceil$. As $R^{k} / G$ is compact, $Q_{k} / G$ is compact (since the function $R^{k} \times[-1,1]^{k} \rightarrow Q_{k}$ mapping $\left(r_{1}, \ldots, r_{k}, \lambda_{1}, \ldots, \lambda_{k}\right)$ to $\lambda_{1} r_{1}+\cdots+\lambda_{k} r_{k}$ is continuous, surjective, and $G$-equivariant). Hence (as $\left.d_{R} \leq d_{2}\right)\left(Q_{k}, d_{R}\right) / G$ is compact, and so $\left(Q_{k}, d_{R} / G\right)$ is compact. Therefore, $Q_{k} \subseteq$ $B_{d_{R} / G}(F, 1 / \sqrt{k})$ for some finite $F$. Then with Proposition 3,

$$
\begin{aligned}
W & \subseteq B(H) \subseteq B_{d_{R}}\left(Q_{k}, 1 / \sqrt{k}\right) \subseteq B_{d_{R} / G}\left(Q_{k}, 1 / \sqrt{k}\right) \\
& \subseteq B_{d_{R} / G}(F, 2 / \sqrt{k}) \subseteq B_{d_{R} / G}(F, \varepsilon) .
\end{aligned}
$$

\subsection{Proof of Theorem 2}

For any graph $F$, define a function

$$
\pi_{F}: \prod_{v \in V(F)} B_{\operatorname{deg}(v)} \rightarrow \mathbb{R} \text { by } \pi_{F}(h):=\sum_{\phi: E(F) \rightarrow C} \prod_{v \in V(F)} h_{v}(\phi(\delta(v)))
$$

for $h=\left(h_{v}\right)_{v \in V(F)} \in \prod_{v \in V(F)} B_{\operatorname{deg}(v)}$. (The sum in (13) converges, as follows from (14) below.)

Proposition 4. If $F$ is a simple graph, then $\pi_{F}$ is continuous on $\prod_{v \in V(F)}\left(B_{\operatorname{deg}(v)}, d_{R_{\operatorname{deg}(v)}}\right)$.

Proof. We first prove the following. For any $k$, any $h \in H_{k}^{S_{k}}$, and any $c_{1}, \ldots, c_{l} \in C$ with $l \leq k$, let $h\left(c_{1}, \ldots, c_{l}\right)$ be the element of $H_{k-l}^{S_{k-l}}$ with $h\left(c_{1}, \ldots, c_{l}\right)\left(c_{l+1}, \ldots, c_{k}\right)=h\left(c_{1}, \ldots, c_{k}\right)$ for all $c_{l+1}, \ldots, c_{k} \in C$. Let $k_{1}, \ldots, k_{n} \in \mathbb{N}$, let $h_{i} \in H_{k_{i}}^{S_{k_{i}}}$ for $i=1, \ldots, n$, and let $F=([n], E)$ be a graph with $\operatorname{deg}(i) \leq k_{i}$ for $i=1, \ldots, n$. Then

$$
\sum_{\phi: E \rightarrow C} \prod_{v \in[n]}\left\|h_{v}(\phi(\delta(v)))\right\| \leq \prod_{v \in[n]}\left\|h_{v}\right\| .
$$

We prove this by induction on $|E|$, the case $E=\emptyset$ being trivial. Let $|E| \geq 1$, and choose an edge $a b \in E$. Set $E^{\prime}:=E \backslash\{a b\}$ and $\delta^{\prime}(v):=\delta(v) \backslash\{a b\}$ for each $v \in V(F)$. Then

$$
\begin{aligned}
& \sum_{\phi: E \rightarrow C} \prod_{v \in[n]}\left\|h_{v}(\phi(\delta(v)))\right\| \\
& =\sum_{\phi: E^{\prime} \rightarrow C} \sum_{c \in C}\left\|h_{a}\left(\phi\left(\delta^{\prime}(a)\right), c\right)\right\|\left\|h_{b}\left(\phi\left(\delta^{\prime}(b)\right), c\right)\right\| \prod_{\substack{v \in[n] \\
v \neq a, b}}\left\|h_{v}(\phi(\delta(v)))\right\| \\
& \leq \sum_{\phi: E^{\prime} \rightarrow C}\left\|h_{a}\left(\phi\left(\delta^{\prime}(a)\right)\right)\right\|\left\|h_{b}\left(\phi\left(\delta^{\prime}(b)\right)\right)\right\| \prod_{\substack{v \in[n] \\
v \neq a, b}}\left\|h_{v}(\phi(\delta(v)))\right\| \leq \prod_{v \in[n]}\left\|h_{v}\right\|,
\end{aligned}
$$

where the inequalities follow from Cauchy-Schwarz and induction, respectively. This proves (14). 
We next prove that for each $h=\left(h_{v}\right)_{v \in V(F)} \in \prod_{v \in V(F)} H_{\operatorname{deg}(v)}$ and each $u \in V(F)$ :

$$
\pi_{F}(h) \leq\left\|h_{u}\right\|_{R_{\operatorname{deg}(u)}} \prod_{\substack{v \in V(F) \\ v \neq u}}\left\|h_{v}\right\| .
$$

To see this, let $N(u)$ be the set of neighbours of $u, F^{\prime}:=F-u$, and $\delta^{\prime}(v):=\delta(v) \backslash \delta(u)$ for $v \in V(F) \backslash\{u\}$. Then

$$
\begin{aligned}
\pi_{F}(h) & =\sum_{\phi: E(F) \rightarrow C} \prod_{v \in V(F)} h_{v}\left(\phi\left(\delta_{F}(v)\right)\right) \\
& =\sum_{\phi: E\left(F^{\prime}\right) \rightarrow C}\left\langle\bigotimes_{v \in N(u)} h_{v}\left(\phi\left(\delta^{\prime}(v)\right)\right), h_{u} \prod_{v \in V\left(F^{\prime}\right) \backslash N(u)} h_{v}(\phi(\delta(v)))\right. \\
& \leq \sum_{\phi: E\left(F^{\prime}\right) \rightarrow C}\left\|h_{u}\right\|_{R_{\operatorname{deg}(u)}} \prod_{v \in V\left(F^{\prime}\right)}\left\|h_{v}\left(\phi\left(\delta^{\prime}(v)\right)\right)\right\| \leq\left\|h_{u}\right\|_{R_{\operatorname{deg}(u)}} \prod_{v \in V\left(F^{\prime}\right)}\left\|h_{v}\right\|,
\end{aligned}
$$

where the inequalities follow from the definition of $\|\cdot\|_{R_{\operatorname{deg}(u)}}$ and from (14) (applied to $F^{\prime}$ ), respectively. This proves (16).

Now let $g, h \in \prod_{v \in V(F)} B_{\operatorname{deg}(v)}$. We can assume that $V(F)=[n]$. For $u=1, \ldots, n$, define $p^{u} \in \prod_{i \in[n]} B_{\operatorname{deg}(i)}$ by $p_{i}^{u}:=g_{i}$ if $i<u, p_{u}^{u}:=g_{u}-h_{u}$, and $p_{i}^{u}:=h_{i}$ if $i>u$. Moreover, for $u=0, \ldots, n$, define $q^{u} \in \prod_{i \in[n]} B_{\operatorname{deg}(i)}$ by $q_{i}^{u}:=g_{i}$ if $i \leq u$ and $q_{i}^{u}=h_{i}$ if $i>u$. So $q^{n}=g$ and $q^{0}=h$. By the multilinearity of $\pi_{F}$ we have $\pi_{F}\left(q^{u}\right)-\pi_{F}\left(q^{u-1}\right)=\pi_{F}\left(p^{u}\right)$. Hence by (16) we have the following, proving the theorem,

$$
\begin{aligned}
\pi_{F}(g)-\pi_{F}(h) & =\sum_{u=1}^{n}\left(\pi_{F}\left(q^{u}\right)-\pi_{F}\left(q^{u-1}\right)\right)=\sum_{u=1}^{n} \pi_{F}\left(p^{u}\right) \leq \sum_{u=1}^{n}\left\|p_{u}^{u}\right\|_{R_{\operatorname{deg}(u)}} \\
& =\sum_{u=1}^{n}\left\|g_{u}-h_{u}\right\|_{R_{\operatorname{deg}(u)}} . \mathbf{\square}
\end{aligned}
$$

Proof of Theorem 2. For each $F \in \mathcal{F}$, the function $\psi: \prod_{k \in \mathbb{N}} B_{k} \rightarrow \prod_{v \in V(F)} B_{\operatorname{deg}(v)}$ mapping $\left(h_{k}\right)_{k \in \mathbb{N}}$ to $\left(h_{\operatorname{deg}(v)}\right)_{v \in V(F)}$ is continuous. As $\pi().(F)=\pi_{F}(\psi()$.$) , the theorem follows from Proposition 4$.

\subsection{Proof of Theorem 3}

We first show:

Proposition 5. Let $\left(X_{1}, \delta_{1}\right),\left(X_{2}, \delta_{2}\right), \ldots$ be complete metric spaces and let $G$ be a group acting on each $X_{k}$, leaving $\delta_{k}$ invariant $(k=1,2, \ldots)$. Then $\left(\prod_{k=1}^{\infty} X_{k}\right) / G$ is compact if and only if $\left(\prod_{k=1}^{t} X_{k}\right) / G$ is compact for each $t$.

Proof. Necessity being direct, we show sufficiency. We can assume that space $X_{k}$ has diameter at most $1 / k$. Let $A:=\prod_{k=1}^{\infty} X_{k}$, and let $d$ be the supremum metric on $A$. Then $d$ is $G$-invariant and $\prod_{k=1}^{\infty}\left(X_{k}, \delta_{k}\right)$ is $G$-homeomorphic with $(A, d)$. So it suffices to show that $(A, d) / G$ is compact.

As each $\left(X_{k}, \delta_{k}\right)$ is complete, $(A, d)$ is complete (cf., e.g., [4, Theorem XIV.2.5]). Hence, by Proposition $2,(A, d / G)$ is complete. So it suffices to prove that $(A, d / G)$ is totally bounded; that is, for each $\varepsilon>0, A$ can be covered by finitely many $d / G$-balls of radius $\varepsilon$.

Set $t:=\left\lfloor\varepsilon^{-1}\right\rfloor$. Let $B:=\prod_{k=1}^{t} X_{k}$ and $C:=\prod_{k=t+1}^{\infty} X_{k}$, with supremum metrics $d_{B}$ and $d_{C}$ respectively. As $B / G$ is compact (by assumption), it can be covered by finitely many $d_{B} / G$-balls of radius $\varepsilon$. As $C$ has diameter at most $1 /(t+1) \leq \varepsilon, A=B \times C$ can be covered by finitely many $d / G$-balls of radius $\varepsilon$. 
Proof of Theorem 3. As each $\left(B_{k}, d_{R_{k}}\right)$ is complete (Proposition 1), by Proposition 5 it suffices to show that for each $t,\left(\prod_{k=0}^{t}\left(B_{k}, d_{R_{k}}\right)\right) / O(H)$ is compact. Consider the Hilbert space $\prod_{k=0}^{t} H_{k}$, and let $W:=\prod_{k=0}^{t} B_{k}$ and $R:=\prod_{k=0}^{t} R_{k}$. Then the identity function on $W$ is a homeomorphism from $\left(W, d_{R}\right)$ to $\prod_{k=0}^{t}\left(B_{k}, d_{R_{k}}\right)$. So it suffices to show that $\left(W, d_{R}\right) / O(H)$ is compact. Now for each $n, R^{n} / O(H)$ is compact, as it is a continuous image of $B(H)^{m} / O(H)$, with $m:=n(1+2+\cdots+t)$. The latter space is compact, as it is a continuous image of the compact space $B\left(\mathbb{R}^{m}\right)^{m}$ (assuming $|C|=\infty$, otherwise $B(H)$ itself is compact). So by Theorem $1,\left(W, d_{R}\right) / O(H)$ is compact.

\section{Acknowledgement}

The research leading to these results has received funding from the European Research Council under the European Union's Seventh Framework Programme (FP7/2007-2013)/ERC grant agreement $\mathrm{n}^{\circ} 339109$.

\section{References}

[1] C. Borgs, J.T. Chayes, L. Lovász, V.T. Sós, K. Vesztergombi, Convergent sequences of dense graphs. I. Subgraph frequencies, metric properties and testing, Adv. Math. 219 (2008) 1801-1851.

[2] P. de la Harpe, V.F.R. Jones, Graph invariants related to statistical mechanical models: examples and problems, J. Combin. Theory Ser. B 57 (1993) 207-227.

[3] J. Draisma, D. Gijswijt, L. Lovász, G. Regts, A. Schrijver, Characterizing partition functions of the vertex model, J. Algebra 350 (2012) 197-206.

[4] J. Dugundji, Topology, Allyn and Bacon, Boston, 1966.

[5] W. Fernandez de la Vega, R. Kannan, M. Karpinski, S. Vempala, Tensor decomposition and approximation schemes for constraint satisfaction problems, in: Proceedings of the 37th Annual ACM Symposium on Theory of Computing, STOC'05, ACM, New York, 2005, pp. 747-754.

[6] A. Frieze, R. Kannan, Quick approximation to matrices and applications, Combinatorica 19 (1999) 175-220.

[7] P.R. Halmos, A Hilbert Space Problem Book, second ed., Springer, New York, 1982.

[8] L. Lovász, Graph homomorphisms: Open problems, Preprint, 2008. http://www.cs.elte.hu/ lovasz/problems.pdf.

[9] L. Lovász, Large Networks and Graph Limits, American Mathematical Society, Providence, RI, 2012.

[10] L. Lovász, B. Szegedy, Limits of dense graph sequences, J. Combin. Theory Ser. B 96 (2006) 933-957.

[11] L. Lovász, B. Szegedy, Szemerédi's lemma for the analyst, Geom. Funct. Anal. 17 (2007) 252-270.

[12] T. Nishiura, Measure-preserving maps of $\mathbb{R}^{n}$, Real Anal. Exchange 24 (1998/9) 837-842.

[13] A. Schrijver, Graph invariants in the edge model, in: M. Grötschel, G.O.H. Katona (Eds.), Building Bridges-Between Mathematics and Computer Science, Springer, Berlin, 2008, pp. 487-498.

[14] B. Szegedy, Edge coloring models and reflection positivity, J. Amer. Math. Soc. 20 (2007) 969-988. 